\title{
Installation of OpenStack (Liberty release) using PackStack
}

\author{
Jaya Tahil Jeswani \\ Assistant Professor, \\ Xavier Institute of Engineering, \\ Mahim (W), Mumbai, India
}

\author{
James Jacob Kurian \\ Student, \\ Xavier Institute of Engineering, \\ Mahim (W), Mumbai, India
}

\author{
John Rose Santiago, PhD \\ Associate Professor, \\ Xavier Institute of Engineering, \\ Mahim (W), Mumbai, India
}

\begin{abstract}
OpenStack is an open source cloud platform created by Rackspace Hosting and NASA. It is primarily used for Infrastructure as a Service (IaaS) deployments. IaaS deployments deal with physical/infrastructural aspects of the virtualization provided by cloud which signifies - storage, computing power, Random Access Memory (RAM) for efficient utilization of resources. This paper presents the installation steps for the 'Liberty' Release of OpenStack using the PackStack installer script. It also describes how to launch an instance using an image of the 'CirrOS' cloud OS, pinging and accessing it via Secure Shell (SSH).
\end{abstract}

\section{Keywords}

Cloud computing, OpenStack, IaaS, Liberty, CirrOS, PackStack, Open Source

\section{INTRODUCTION}

Over the last decade cloud computing $[1,2,4]$ has grown from being a promising business concept to one of the fastest growing segments of the IT industry. Big companies like Amazon, Google, Microsoft etc., expand their market by adopting Cloud Computing systems which enhance their services provided to a large number of users. The term Cloud computing refers to the delivery of computing as a service rather than a product. Below figure 1 shows various cloud services.

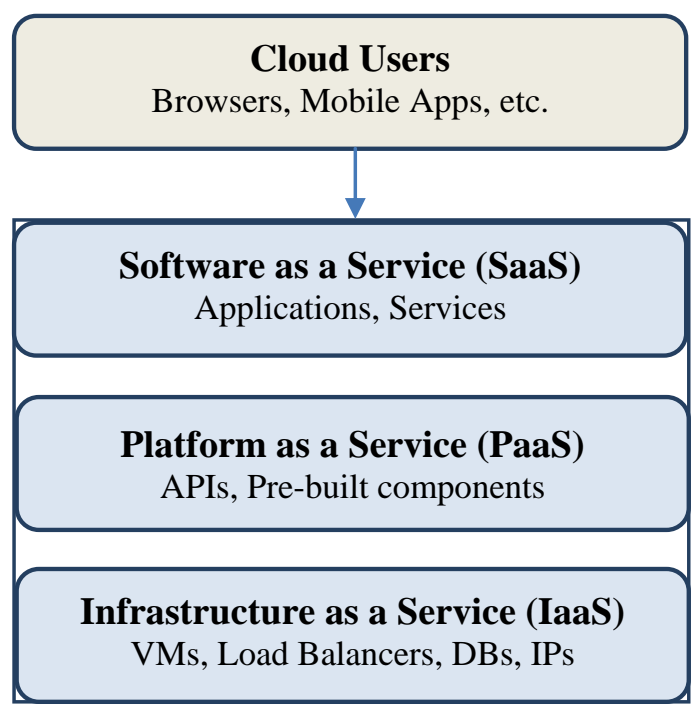

Fig. 1: Cloud layered model

Furthermore, it is a design concept which tries to separate the application from the operating system on which the hardware runs. Users are charged based on short-term basis. Cloud computing is scalable in nature. It provides various services like Data as a Service (DaaS), Software as a Service (SaaS), Platform as a Service (PaaS) or Infrastructure as a Service (IaaS). Therefore, one can say that Cloud Computing has evolved at an incredible pace.

In literature, Anshu [5] has compared various OpenStack installers. Rakesh [8] has described various solutions for deploying cloud environments with a special emphasis on OpenStack- installation and components. Rohit [4] has described various components of OpenStack.

The organization of the paper is as follows: In section 2 OpenStack is explained. Section 3 describes the prerequisites for installation and implementation steps of the Liberty release of OpenStack whereas in section 4 experimental results are discussed.

\section{OPENSTACK}

OpenStack [1] is a collection of software components that enable one to leverage the power of resources like computing power, memory, and other specialized storage structures like object storage and block storage distributed across datacentres. OpenStack consists of a set of continually advancing but mature components specializing in various aspects of cloud computing as mentioned in Table 1 .

Table 1: Some of the OpenStack components/services

\begin{tabular}{|c|c|}
\hline Service Name & Service Provided \\
\hline Horizon & Dashboard \\
\hline Ceilometer & Telemetry \\
\hline Nova & Compute \\
\hline Neutron & Networking \\
\hline Swift & Object Storage \\
\hline Cinder & Block Storage \\
\hline Keystone & Identity \\
\hline Glance & Image Service \\
\hline
\end{tabular}

Various OpenStack components/services can be combined to create various configurations which can include a general compute grid or Big Data storage and processing clusters. OpenStack is driven by community efforts and backed by RackSpace Cloud Computing. Community gatherings or summits tend to involve many other ideas fuelling the growth of the OpenStack cloud ecosystem. 


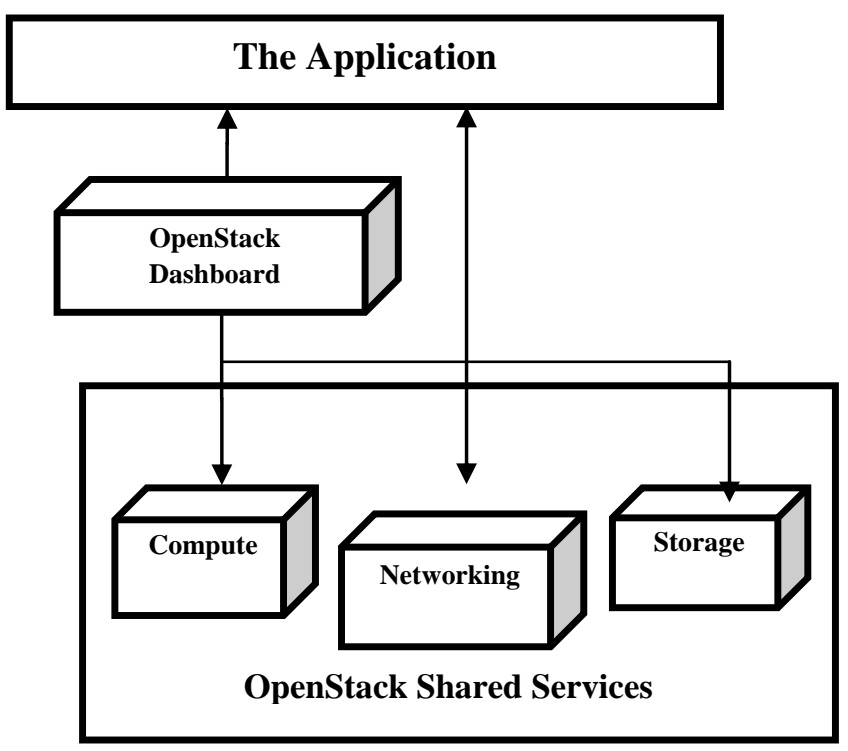

Fig. 2: OpenStack services

As shown in the figure2, OpenStack primarily consists of Compute (Nova), Networking (Neutron) and Storage (Swift, Cinder, Glance) components. The OpenStack Dashboard (Horizon) provides a graphical user interface to control the various services of OpenStack without having to resort to executing commands on a terminal/console. Thus, the UI provides a way to control and manage resources in a user friendly manner thereby eliminating the need for technical experts for doing simple tasks. At the same time, OpenStack gives a lot of power to developers by providing a rich API using which various services can be controlled and managed. This means scalability and extensibility become a lot easier.

OpenStack releases are named in a similar fashion as Android Operating Systems (i.e. in an alphabetical order).

Table 2: OpenStack Releases (Latest first)

\begin{tabular}{|c|c|c|c|}
\hline Sr. No & Name & Year & Month \\
\hline 1 & Liberty & 2015 & Oct \\
\hline 2 & Kilo & 2015 & Apr \\
\hline 3 & Juno & 2014 & Oct \\
\hline 4 & Icehouse & 2014 & Apr \\
\hline 5 & Havana & 2013 & Oct \\
\hline 6 & Grizzly & 2013 & Apr \\
\hline 7 & Folsom & 2012 & Apr \\
\hline 8 & Essex & 2012 & Apr \\
\hline 9 & Diablo & 2011 & Sep \\
\hline 10 & Cactus & 2011 & Apr \\
\hline 11 & Bexar & 2011 & Feb \\
\hline 12 & Austin & 2010 & Oct \\
\hline
\end{tabular}

PackStack is a script that installs various components of OpenStack in one go. Rather than installing and configuring each component separately, the PackStack script allows one to install and pre-configure a subset of the components by executing a single command.

\section{PROPOSED METHOD}

Proposed method section is divided into three sub-sections. Sub-section 3.1 gives pre-requisites for OpenStack installation using PackStack. Sub-section 3.2 shows a flowchart for installation steps of OpenStack. Sub-section 3.3 gives implementation steps of OpenStack's 'Liberty' release.

\subsection{Pre-requisites}

Installation can be done on a virtual machine or on a bare minimum system. If VMware Workstation is being used, check the checkboxes labeled 'Virtualize Intel VT-x/EPT or AMD-V/RVI' and 'Virtualize CPU performance counters'.

\subsection{Flowchart of installation steps of OpenStack using PackStack}

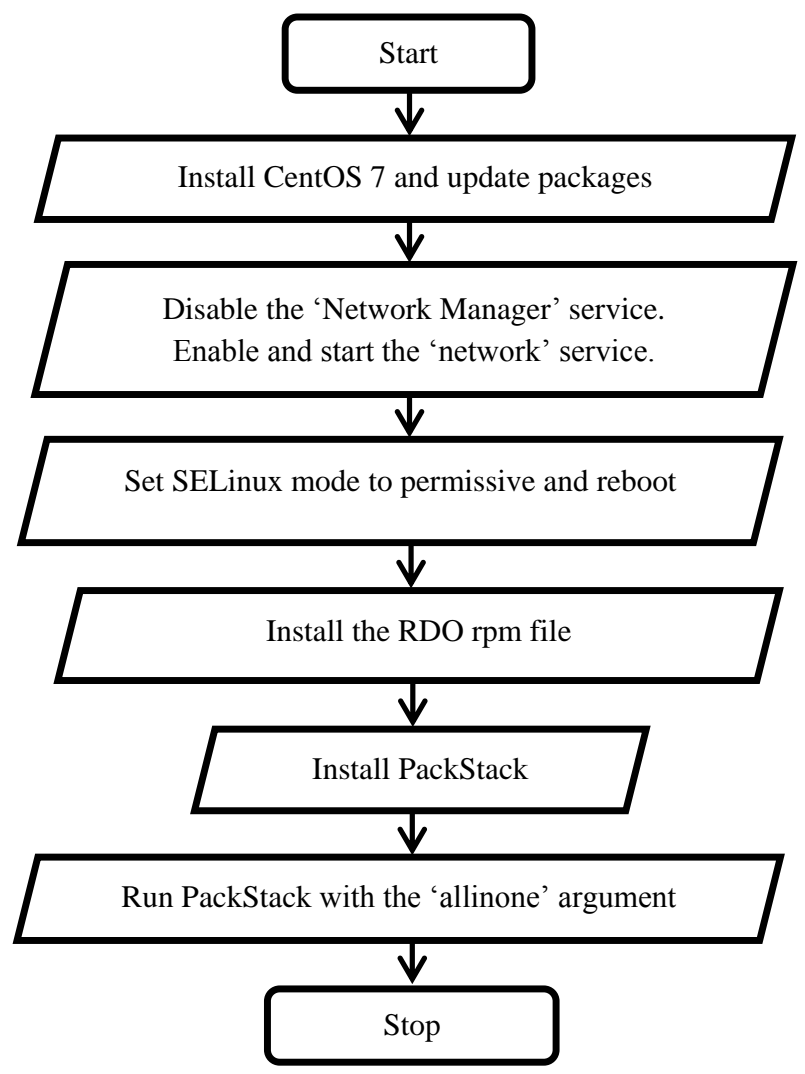

Fig. 3: Flowchart depicting installation steps of OpenStack

\subsection{Implementation Steps of Latest Release of OpenStack (Liberty)}

1. Install CentOS and update packages: sudo yum update

2. Disable the 'Network Manager' service. Enable and start the 'network' service instead.

3. Set SELinux to permissive mode (figure4)

4. Reboot

5. Install the RDO rpm file:sudo yum install $-\mathrm{y}$ https://www.rdoproject.org/repos/rdo-release.rpm

6. Install PackStack:sudo yum install openstack-packstack

7. Run PackStack with the 'allinone' argument: packstack -allinone

\section{EXPERIMENTAL RESULTS}

Experimental results section is divided into four sub-sections. Sub-section 4.1 shows various screenshots for OpenStack installation using PackStack. Sub-section 4.2 shows screenshots of launching a CirrOS instance. Sub-section 4.3 associates an IP address with an instance. Sub-section 4.4 shows screenshot of logging into an instance using SSH with a private key and getting root privileges. 


\subsection{Dashboard installation}

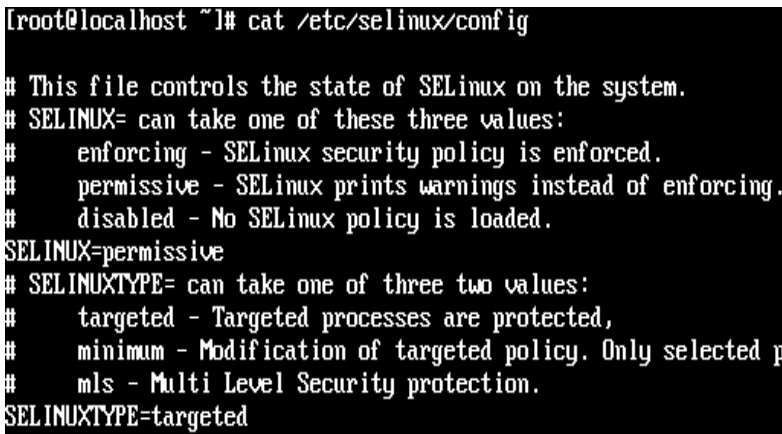

Fig. 4: Setting SELinux to permissive mode

Figure 4 shows SELinux set to permissive mode so that it doesn't interfere with the installation of various OpenStack components as the PackStack script changes various settings which SELinux might not allow.

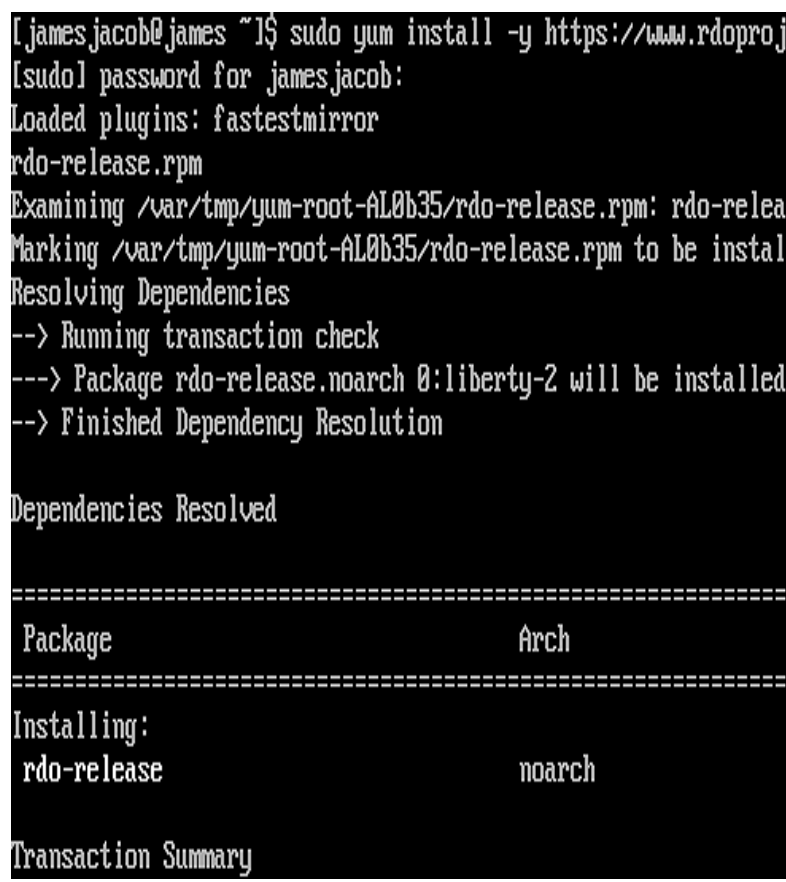

Fig. 5: Installing the RDO rpm file

Figure 5 shows installation of the RDO (RPM Distribution of OpenStack) rpm file.

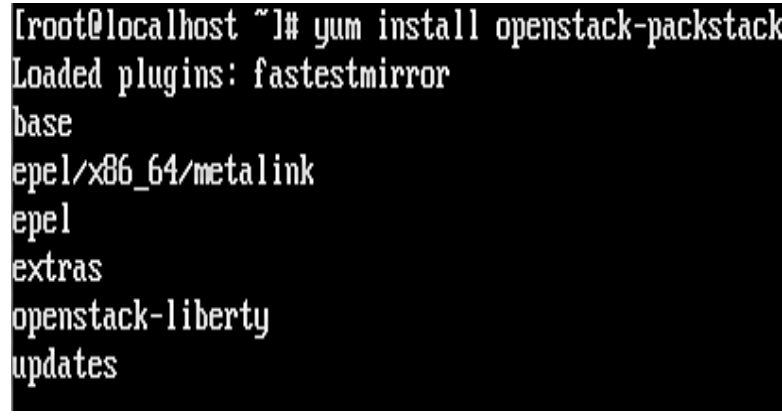

Fig. 6: Installing the PackStack script

Figure 6 shows the beginning of the installation of the PackStack script.
Below figure 7 shows the start of the execution of the PackStack script with the 'allinone' switch enabled.

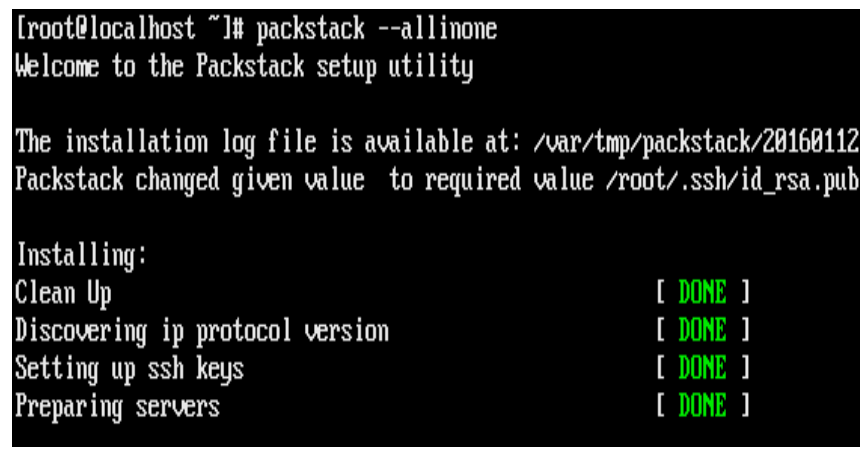

Fig. 7: Running the PackStack script with the 'allinone' switch enabled

On successful execution of the PackStack script, details regarding the Horizon and Keystone URIs and default users and login password are printed on the screen.

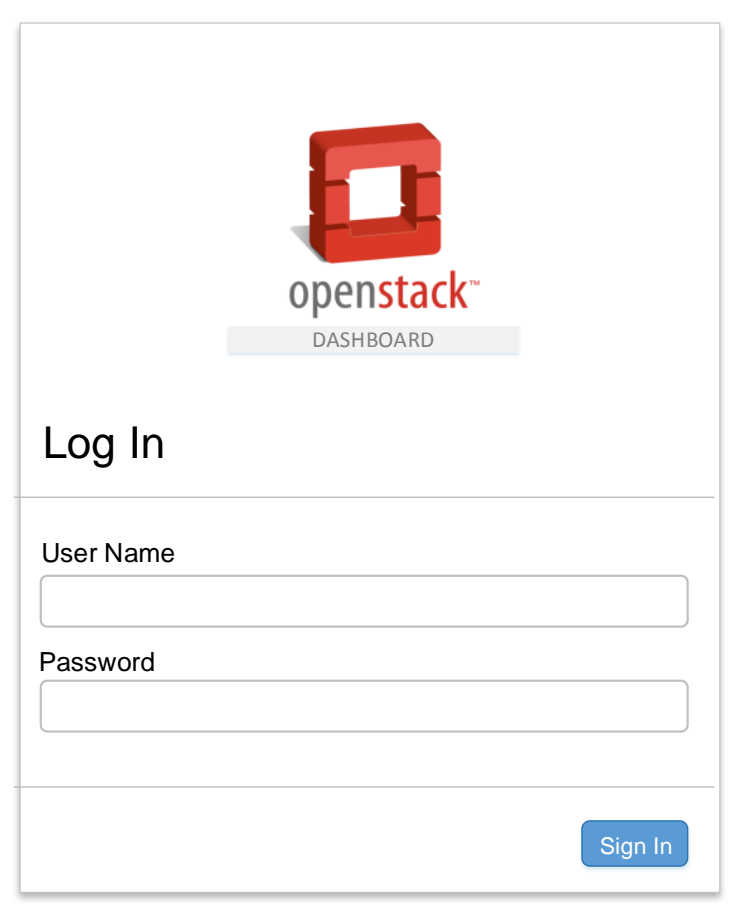

Fig. 8: OpenStack dashboard (Horizon)

Figure 8 shows a screenshot of the OpenStack dashboard (Horizon) as it appears in the Mozilla Firefox Browser.

\subsection{Launching a CirrOS instance}

On successful login to the dashboard, one is presented with a screen that shows various components.

1. Click on 'Project'.

2. Then click on 'Instances' under the 'Compute' menu.

3. Click on 'Launch Instance'.

4. Enter the amount of computing resources along with the image name (figure9). 


Details * Access \& Security
Availability Zone
nova
Instance Name *
JJinstance
Instance ${ }^{*}$ -
m1.nano
Instance Boot Source ${ }^{*}$ O
Boot from image
Image Name ${ }^{*}$
cirros-0.3.4-x86_64-uec $(24.0 \mathrm{MB})$

Advanced Options

Specify the details for launching an instance.

The chart below shows the resources used by this project in relation to the project's quotas.

Flavor Details

$\begin{array}{ll}\text { Name } & \mathrm{m} 1 \text {.nano } \\ \text { vCPUs } & 1 \\ \text { Root Disk } & 0 \mathrm{~GB} \\ \text { Ephemeral Disk } & 0 \mathrm{~GB} \\ \text { Total Disk } & 0 \mathrm{~GB} \\ \text { RAM } & 64 \mathrm{MB}\end{array}$

Project Limits

Number of Instances $\quad 0$ of 10 Used

Number of VCPUs $\quad 0$ of 20 Used

Dotal RAM -

Total RAM $\quad 0$ of 51,200 MB Used

Totaram

Fig. 9: Launching an instance - Details screen

5. Assign a new key pair by clicking on the plus (+) symbol.

To generate a key pair, type:

ssh- keygen - $\mathrm{t}$ rsa $-\mathrm{f}$ <name_of_key.key>

Two keys will be generated - one public key and one private key as shown in figure 10 .

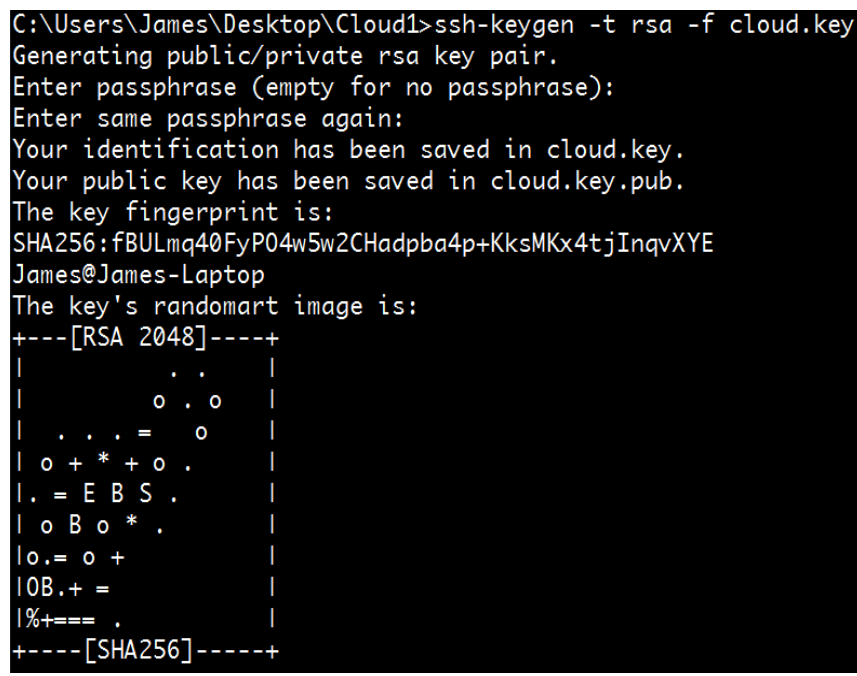

Fig. 10: Generating an RSA key pair

SSH uses the RSA (Rivest Shamir Adleman) method which generates public and private keys. The private key is to be kept safely by the user who wishes to log in to the instance.

6. Copy the contents of the public key file and paste it into the 'Public Key' field as shown in figure 11.
Import Key Pair

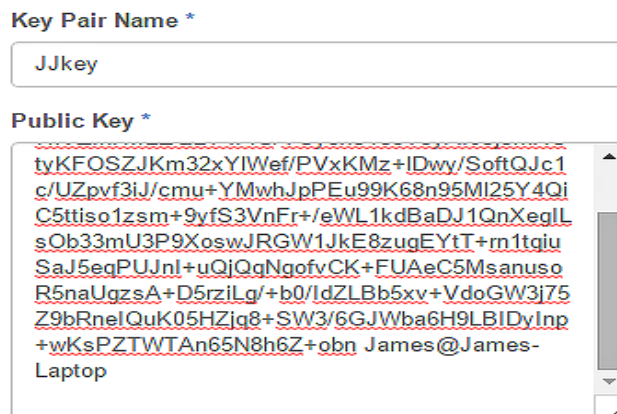

\section{Description:}

Key Pairs are how you login to your instance after it is launched.

Choose a key pair name you will recognise and paste your SSH public key into the space provided.

SSH key pairs can be generated with the ssh-keygen command:

ssh-keygen -t rsa -f cloud.key

This generates a pair of keys: a key you keep private (cloud.key) and a public key (cloud.key.pub). Paste the contents of the public key file here.

After launching an instance, you login using the private key (the username might be different depending on the image you launched):

ssh -i cloud.key <username>e<instance_ip>

Cancel

Import Key Pair

Fig. 11: Import key pair

Below figure 12 shows a screenshot of associating a key with the instance. 
Launch Instance

Details * Access \& Security
Key Pair $\boldsymbol{0}$

Fig. 12: Launching an instance - Associating a key with the instance

7. Additionally, one can specify CloudInit data (figure 13) to boot the instance with data that will automatically run when the instance is started.

Launch Instance

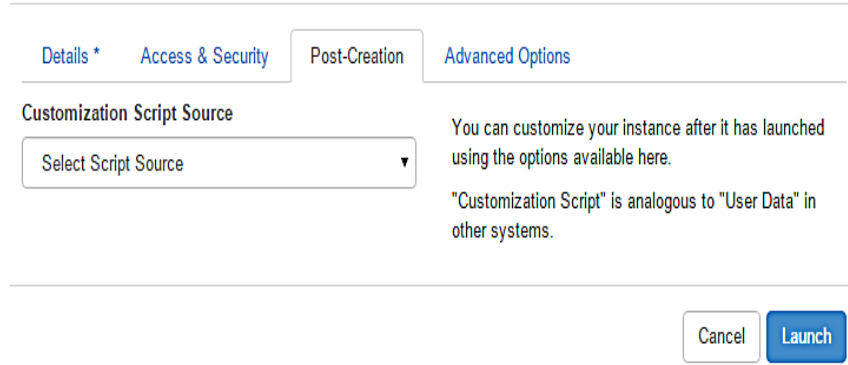

Fig. 13: Launching an instance - CloudInit data input

8. Disk partitioning can also be done at this stage as shown in the figure 13 .

\section{Launch Instance}

\begin{tabular}{lll}
\hline Details * Access \& Security & Post-Creation & Advanced Options \\
\hline Disk Partition 0 & $\begin{array}{l}\text { Specify advanced options to use when launching an } \\
\text { instance. }\end{array}$ \\
\hline Automatic & \\
\hline Configuration Drive 0 &
\end{tabular}

Fig. 14: Launching an instance -Disk partitioning screen

9. Click on 'Launch' to launch the instance. The new instance will be listed in the 'Instances' section. The instance will start spawning. After some time, the instance will have launched successfully if the required resources are available.

\section{Instances}

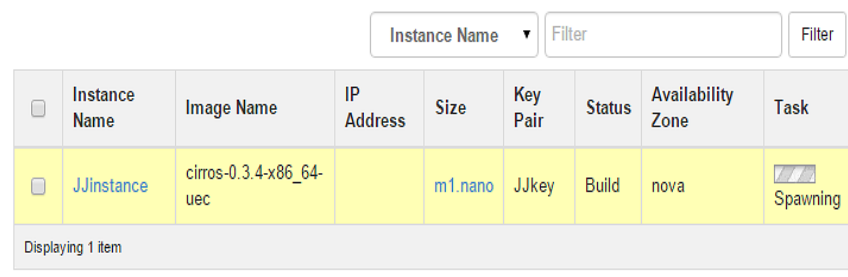

Fig. 15: Launching and instance - Spawning

The above figure 15 shows the instance as it is spawning.

CirrOS is a cloud operating system and is downloaded and bundled by default when one runs the PackStack script. It doesn't have all the capabilities of a full-fledged operating system like CentOS and Ubuntu. For example, installers like yum and sudo are not available on a fresh installation of CirrOS which are, by default, present in most of the distributions of Linux. As CirrOS is bundled, it will appear in the list of images automatically.

\subsection{Pinging the IP address of the instance}

Once the instance has been launched one can ping the instance to ensure that connectivity has been established. Assign a floating IP to the instance from the 'actions' menu so that it can be accessed from a public network.

$$
\begin{aligned}
& \text { Associate Floating IP } \\
& \text { Disassociate Floating IP } \\
& \text { Edit Instance } \\
& \text { Edit Security Groups }
\end{aligned}
$$

Fig. 16: Associating a floating IP address to an instance

Figure 16 shows a dropdown menu which lists various options which can be used to control instance behavior.

ping <ip_address>

If the packets loss is zero or negligible then connectivity has been established.

\subsection{Using SSH to login into the instance as root user}

$\mathrm{SSH}$ access is also possible. Once it is determined whether the instance can be successfully pinged to, SSH can be used to log into the instance.

The preferred way of logging into an instance using SSH is using keys that were previously generated during the creation of the instance. This is because the user who wishes to log in needs the private key during the connection step. If the key fails, the user will be prompted for a password. But the key adds one layer of security.

Without a key:

ssh<username>@<ip_address>

With a key

ssh -i <keyname>.key <username>@ @ip_address>

Once one has logged in to the instance successfully, one can get root privileges by using the sudo command:

sudo $\mathrm{su}-$ 


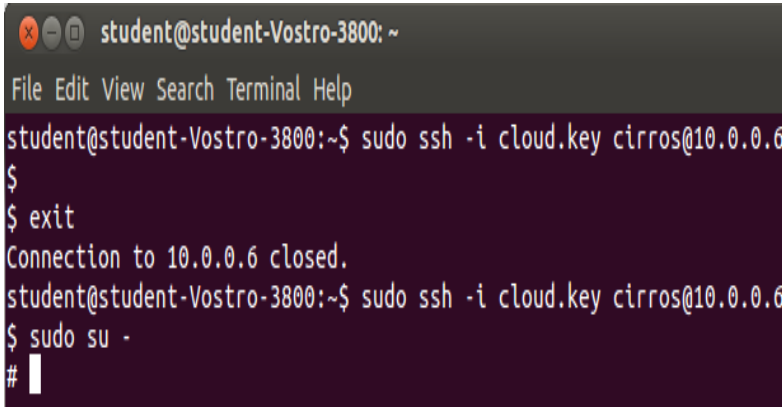

Fig. 17: Logging into an instance using SSH with a private key and getting root privileges

Figure 17 shows the use of SSH to log into an instance using the supplied private key. On logging in, the command 'sudo su -', was used to get root privileges.

\section{APPENDIX}

The paper recommends having more than 4 GB RAM, CPU with virtualization capability, hard drive with capacity greater than 100GB and an active Internet connection. Also, an ISO image of the operating system will be required. The paper assumes a 64-bit CentOS7 as the base operating system. If using a hypervisor ensure that Intel VT-x or AMD-V are enabled.

\section{FUTURE WORK}

Various OpenStack components can be worked on, say, the network components like Neutron, storage components like Swift and Cinder, identity components like Keystone, compute components like Nova, etc. Load balancing and security are areas that can be explored. With each new release of OpenStack, various components and options could be added which would give more flexibility to deploy clouds.

\section{CONCLUSION}

The paper describes a method for installation of OpenStack using the PackStack script. The script fetches various components from the required repositories and sets them up automatically. PackStack simplifies installation of various OpenStack components by pre-configuring various subcomponents required for running various OpenStack services. Instances launched can be accessed via SSH which was also described in the paper. The requirements and configuration setup described in this paper are cost effective and deployment can be done on inexpensive hardware at the time of this writing.

\section{REFERENCES}

[1] Jiang Yunxia, Zhao Bowen, Wang Shuqi and Sun Dongnan, "Research of Enterprise Private Cloud Computing Platform Based on OpenStack," International Journal of Grid Distribution Computing, Vol.7, No.5, pp. no. 171-180, 2014

[2] Vamsi Krishna Pelluru, "Storage Area Networks with OPENSTACK and Installation of Havana," International Journal of Inventive Engineering and Sciences (IJIES), Vol. 2, Issue 4, March 2014.

[3] Dr. Urmila R. Pol, "Developers Harness Open Source Cloud Management Platforms for Novel Applications," International Journal of Computer Science and Information Technologies, Vol. 5, 6483-6486, 2014.

[4] Rohit Kamboj and Anoopa Arya, "OpenStack: Open Source Cloud Computing IaaS Platform," International
Journal of Advanced Research in Computer Science and Software Engineering, Vol. 4, Issue 5, May 2014.

[5] Anshu Awasthi and Ravi Gupta P, "Comparison of OpenStack Installers," International Journal of Innovative Science, Engineering \& Technology, Vol. 2, Issue 9, September 2015.

[6] Dr. Deepak Arora, Varun Kumar, B. Jagdeep, Prabhat Verma, "Proposed Model for Virtual Labs Interaction with OpenStack Integration Using KVM Hypervisor," International Journal of Scientific \& Technology Research, Vol. 3, Issue 7, July 2014.

[7] Omar, Mohammed, Mohsine, "OpenStack: Toward an Open-Source Solution for Cloud Computing," International Journal of Computer Applications, Vol. 55, No. 03, pg. no. 0975 - 8887, October 2012.

[8] Rakesh Kumar, Neha Gupta, Shilpi Charu, Kanishk Jain, Sunil Kumar Jangir, "Open Source Solution for Cloud Computing Platform Using OpenStack," International Journal of Computer Science and Mobile Computing, Vol. 3, Issue 5, pg. 89-98, May 2014

[9] Rdoproject.org, "RDO Quickstart," Online. Available: https://www.rdoproject.org/install/quickstart. Accessed on 1 January 2016.

[10] Sans.org, "SANS Institute InfoSec Reading Room," [Whitepaper]. Available: https://www.sans.org/readingroom/whitepapers/linux/intr oduction-nsas-security-enhanced-linux-selinux-232. Accessed on 10 January 2016.

[11] Zoran Pantic and Muhammad Ali Babar, "Guidelines for building a Private Cloud Infrastructure," Tech Report, IT University of Copenhagen, 2012.

[12] Docs.openstack.org, “OpenStack Cloud Administrator Guide," [Online]. Available: http://docs.openstack.org/admin-guide-cloud/. Accessed on 1 January 2015.

[13] OpenStack.org, "OpenStack User Stories," [Online]. Available: http://www.openstack.org/user-stories/. Accessed on 20 January 2016.

[14] News.slashdot.org, "Is AT\&T Building the Ultimate Walled Garden?," [Online]. Available http://news.slashdot.org/story/12/01/11/232234/is-attbuilding-the-ultimate-walled-garden. Accessed on 20 January 2016.

[15] Youtube.com, "BBVA Bank on OpenStack," [Online]. Available:

https://www.youtube.com/watch?v=PESWFDPbexs. Accessed on 20 January 2016.

[16] Dailycloud.info, "Box Deploys Platform9 In Key Win For OpenStack," [Online]. Available: http://www.dailycloud.info/box-deploys-platform9-inkey-win-for-openstack. Accessed on 20 January 2016.

[17] Openstack.org, "Releases," [Online]. Available: https://wiki.OpenStack.org/wiki/Releases. Accessed on 1 January 2016.

[18] Openstack.org, "Software," [Online]. Available: https://www.OpenStack.org/software/. Accessed on 1 January 2016. 